\title{
ARITHMETICAL FUNCTIONS OF A GREATEST COMMON DIVISOR. I
}

\section{ECKFORD COHEN}

1. Introduction. Let $m$ and $n$ denote positive integral variables. Also place

$$
f_{\alpha}(n)=\sum_{d \delta=n} g(d) \delta^{\alpha},
$$

where $g(n)$ is a bounded arithmetical function and $\alpha$ a real number. In this paper we investigate the average order of magnitude of functions of the form $f_{\alpha}((m, n))$ where $(m, n)$ denotes the greatest common divisor of $m$ and $n$ and $\alpha \geqq 1$. The principal result is embodied in the theorem of $\$ 3$.

The method of the paper is elementary. It is based upon an identity (Lemma 3.1) which enables us to reduce the problem in question to the consideration of arithmetical functions in a single variable. The only other tools required are some auxiliary identities and several elementary estimates, all contained in $\$ 2$.

Of particular interest are the functions (1.1) which arise when $g(n)=1$ and $g(n)=\mu(n)$, where $\mu(n)$ denotes the Möbius function. In the first case, $f_{\alpha}(n)$ becomes the generalized divisor function $\sigma_{\alpha}(n)$, while in the second case $f_{\alpha}(n)$ reduces to the generalized totient $\phi_{\alpha}(n)$ :

$$
\sigma_{\alpha}(n)=\sum_{d \delta=n} \delta^{\alpha}, \quad \phi_{\alpha}(n)=\sum_{d \delta=n} \mu(d) \delta^{\alpha} .
$$

Following the usual practice, we write $\sigma(n)=\sigma_{1}(n), \phi(n)=\phi_{1}(n)$. The corollaries of $\S 3$ are devoted to estimates for the functions $\sigma_{\alpha}((m, n))$ and $\phi_{\alpha}((m, n)), \alpha \geqq 1$. We mention that the functions $\sigma((m, n))$ and $\sigma_{2}((m, n))$ were considered originally by Cesàro [2].

2. Identities and estimates involving functions of a single variable. In this paper $x$ will be assumed real and $\geqq 2$. The summatory function $G(x)$ of an arithmetical function $g(n)$ is defined by

$$
G(x)=\sum_{n \leqq x} g(n) .
$$

In the first two lemmas of this section $f(n)$ will be defined by

Presented to the Society, November 29, 1958 under the title Asymptotic averages on a class of arithmetical functions of two variables. I; received by the editors April 8 , 1959. 


$$
f(n)=\sum_{d \delta=n} g(d) h(\delta),
$$

where $h(n)$ is an arithmetical function with summatory function $H(x)$.

The following two results are known.

Lemma 2.1. If $f(n)$ is defined by (2.1), then

$$
\sum_{n \leq x} f(n)=\sum_{n \leq x} g(n) H\left(\frac{x}{n}\right)=\sum_{n \leq x} h(n) G\left(\frac{x}{n}\right) .
$$

Proof. We have

$$
\sum_{n \leq x} f(n)=\sum_{n \leq x} \sum_{d \delta=n} g(d) h(\delta)=\sum_{d \leq x} g(d) \sum_{\delta \leq x / d} h(\delta),
$$

which proves the equality of the first and second expressions in (2.2). The equality of the first and third is proved similarly.

Lemma 2.2 (Compare [4, (4); 3, p. 317]). If $f(n)$ is defined by (2.1), then for all $\delta_{1}, \delta_{2}$ satisfying $0<\delta_{1} \leqq x, 0<\delta_{2} \leqq x, \delta_{1} \delta_{2}=x$,

$$
\sum_{n \leq x} f(n)=\sum_{n \leq \delta_{1}} g(n) H\left(\frac{x}{n}\right)+\sum_{n \leq \delta_{2}} h(n) G\left(\frac{x}{n}\right)-G\left(\delta_{1}\right) H\left(\delta_{2}\right) .
$$

Proof. By (2.2) one may write

$$
\sum_{n \leq x} f(n)=\sum_{n \leq \delta_{1}} g(n) H\left(\frac{x}{n}\right)+\sum_{\delta_{1}<n \leq x} g(n) H\left(\frac{x}{n}\right) \equiv \sum_{1}+\sum_{2} ;
$$

moreover,

$$
\begin{aligned}
\sum_{2} & =\sum_{\delta_{1}<n \leqq x} g(n) \sum_{a \leqq x / n} h(a)=\sum_{a \leqq \delta_{2}} h(a) \sum_{\delta_{1}<n \leqq x / a} g(n) \\
& =\sum_{a \leqq \delta_{2}} h(a) G\left(\frac{x}{a}\right)-G\left(\delta_{1}\right) H\left(\delta_{2}\right),
\end{aligned}
$$

and (2.3) is proved.

The following classical elementary estimates, needed in the later discussion, will be cited without proof.

$$
\begin{array}{rlr}
\sum_{n>x} \frac{1}{n^{\alpha}} & \equiv \zeta(\alpha)-\sum_{n \leq x} \frac{1}{n^{\alpha}}=O\left(\frac{1}{x^{\alpha-1}}\right) & \text { if } \alpha>1 ; \\
\sum_{n>x} \frac{\log n}{n^{2}} & =O\left(\frac{\log x}{x}\right) ; & \text { if } \alpha \geqq 0, \\
\sum_{n \leq x} n^{\alpha} & =\frac{x^{\alpha+1}}{\alpha+1}+\left\{\begin{array}{lr}
O\left(x^{\alpha}\right) & \text { if }-1<\alpha<0 ; \\
O(1) & <-\alpha<
\end{array}\right.
\end{array}
$$




$$
\sum_{n \unlhd x} \frac{1}{n}=\log x+\gamma+o\left(\frac{1}{x}\right),
$$

where $\gamma$ is Euler's constant.

Define $L(s, g)$ by the Dirichlet series,

$$
L(s, g)=\sum_{n=1}^{\infty} \frac{g(n)}{n^{s}}
$$

and let $L^{\prime}(s, g)$ denote the derivative of $L(s, g)$.

Lemma 2.3. Let $g(n)$ be bounded. Then if $\alpha>0$,

$$
\sum_{n \leqq x} f_{\alpha}(n)=\left(\frac{L(\alpha+1, g)}{\alpha+1}\right) x^{\alpha+1}+O\left(e_{\alpha}(x)\right),
$$

where $e_{\alpha}(x)=x^{\alpha}, x \log x$, or $x$ according as $\alpha>1, \alpha=1$, or $\alpha<1$;

$$
\begin{gathered}
\sum_{n \leqq x} \frac{f_{1}(n)}{n^{2}}=L(2, g)(\log x+\gamma)+L^{\prime}(2, g)+O\left(\frac{\log x}{x}\right), \\
\sum_{n \leqq x} \frac{f_{1}(n)}{n}=O(x) .
\end{gathered}
$$

Proof. In all cases

$$
\sum_{n \leqq x} \frac{f_{\alpha}(n)}{n^{t}}=\sum_{n \leq x} \frac{1}{n^{t}} \sum_{d \delta=n} g(d) \delta^{\alpha}=\sum_{d \leqq x} \frac{g(d)}{d^{t}} \sum_{\delta \leqq x / d} \delta^{\alpha-t} .
$$

CASE $1(\alpha>0, t=0)$. In this case (2.11) yields, in conjunction with (2.6),

$$
\begin{aligned}
\sum_{n \leq x} f_{\alpha}(n) & =\frac{x^{\alpha+1}}{\alpha+1} \sum_{d \leqq x} \frac{g(d)}{d^{\alpha+1}}+O\left(x^{\alpha} \sum_{d \leqq x} \frac{1}{d^{\alpha}}\right) \\
& =\left(\frac{L(\alpha+1, g)}{\alpha+1}\right) x^{\alpha+1}+O\left(x^{\alpha+1} \sum_{d>x} \frac{1}{d^{\alpha+1}}\right)+O\left(e_{\alpha}(x)\right),
\end{aligned}
$$

and (2.8) follows by (2.4).

CASE $2(\alpha=1, t=2)$. In this case, (2.11) becomes, by virtue of (2.7),

$$
\begin{aligned}
\sum_{n \leq x} \frac{f_{1}(n)}{n^{2}}= & (\log x+\gamma) \sum_{d \leq x} \frac{g(d)}{d^{2}}-\sum_{d \leq x} \frac{g(d) \log d}{d^{2}}+O\left(\frac{1}{x} \sum_{d \leq x} \frac{1}{d}\right) \\
= & (\log x+\gamma) L(2, g)+L^{\prime}(2, g) \\
& +O\left(\log x \sum_{d>x} \frac{1}{d^{2}}\right)+O\left(\sum_{d>x} \frac{\log d}{d^{2}}\right)+O\left(\frac{\log x}{x}\right),
\end{aligned}
$$


and (2.9) results on the basis of (2.4) and (2.5).

CASE $3(\alpha=t=1)$. In this case $(2.10)$ follows immediately from (2.11) since $g(n)$ is bounded. The lemma is proved.

Recalling the familiar fact, $\zeta^{-1}(s)=\sum_{n=1}^{\infty} \mu(n) n^{-s}(s>1)$, we note that the case $\alpha=1, g(n)=\mu(n)$ in (2.8) yields the classical estimate of Mertens for the average order of $\phi(n)$,

$$
\Phi(x) \equiv \sum_{n \leqq x} \phi(n)=\frac{x^{2}}{2 \zeta(2)}+O(x \log x) .
$$

We prove the following additional estimates for $\phi(n)$.

LEMma 2.4. If $t>0$, then

$$
\sum_{n \leq x} \frac{\phi(n)}{n^{t}}= \begin{cases}\frac{\zeta(t-1)}{\zeta(t)}+O\left(\frac{1}{x^{t-2}}\right) & \text { if } t>2 \\ \frac{1}{\zeta(t)}\left(\log x-\frac{\zeta^{\prime}(2)}{\zeta(2)}+\gamma\right)+O\left(\frac{\log x}{x}\right) & \text { if } t=2 \\ O\left(x^{2-t}\right) & \text { if } t<2\end{cases}
$$

Proof. The case $t<2$ follows by (2.6) in view of the fact, $\phi(n) \leqq n$. The case $t=2$ is the case $g(n)=\mu(n)$ in (2.9). In case $t>2$, we have, on the basis of (2.11), with $g(n)=\mu(n), \alpha=1$, in connection with (2.4),

$$
\begin{aligned}
\sum_{n \leq x} \frac{\phi(n)}{n^{t}} & =\zeta(t-1) \sum_{d \leq x} \frac{\mu(d)}{d^{t}}+O\left(x^{2-t} \sum_{d \leq x} \frac{1}{d^{2}}\right) \\
& =\frac{\zeta(t-1)}{\zeta(t)}+O\left(\sum_{d>x} \frac{1}{d^{t}}\right)+O\left(x^{2-t}\right)=\frac{\zeta(t-1)}{\zeta(t)}+O\left(x^{2-t}\right) .
\end{aligned}
$$

The lemma is proved.

3. The average order of $f_{\alpha}((m, n))$. Let $\Phi(x)$ denote the summatory function of $\phi(n)$. The following lemma is basic in the proof of the main result of this section.

Lemma 3.1. If $f(n)$ is an arbitrary arithmetical function, then

$$
\sum_{a, b \leqq x} f((a, b))=2 \sum_{n \leqq x} f(n) \Phi\left(\frac{x}{n}\right)-\sum_{n \leqq x} f(n) .
$$

Proof. Let $Q(x)$ denote the number of ordered pairs of positive integers $a, b \leqq x$ such that $(a, b)=1$. Then clearly

$$
\sum_{a, b \leq x} f((a, b))=\sum_{n \leqq x} Q\left(\frac{x}{n}\right) f(n) .
$$


But it is easily observed $[1$, p. $430 ; 5]$ that $Q(x)=2 \Phi(x)-1$. Hence (3.1) follows immediately.

We are now ready to prove our

Theorem. Let $g(n)$ be bounded. Then in case $\alpha>1$,

$$
\begin{aligned}
& \sum_{a, b \leq x} f_{\alpha}((a, b)) \\
& \quad=\frac{L(\alpha+1, g)}{(\alpha+1) \zeta(\alpha+1)}(2 \zeta(\alpha)-\zeta(\alpha+1)) x^{\alpha+1}+O\left(E_{\alpha}(x)\right),
\end{aligned}
$$

where

$$
E_{\alpha}(x)= \begin{cases}x^{\alpha} & (\alpha>2), \\ x^{2} \log x & (\alpha=2), \\ x^{2} & (1<\alpha<2)\end{cases}
$$

in case $\alpha=1$,

$$
\begin{aligned}
& \sum_{a, b \leq x} f_{1}((a, b)) \\
(3.4)= & \frac{x}{\zeta(2)}\left\{L(2, g)\left(\log x+2 \gamma-\frac{1}{2}-\frac{\zeta(2)}{2}-\frac{\zeta^{\prime}(2)}{\zeta(2)}\right)+L^{\prime}(2, g)\right\} \\
& +O\left(x^{3 / 2} \log x\right) .
\end{aligned}
$$

Proof. Let $F_{\alpha}(x)$ denote the summatory function of $f_{\alpha}(n)$. For all $\alpha \geqq 1$, it follows by Lemma 3.1 that

$$
\sum_{a, b \leq x} f_{\alpha}((a, b))=2 \sum_{n \leq x} f_{\alpha}(n) \Phi\left(\frac{x}{n}\right)-F_{\alpha}(x) \equiv 2 \sum_{1}^{\alpha}-\sum_{2}^{\alpha} .
$$

We now separate the proof into two parts according as $\alpha>1$ or $\alpha=1$.

CASE $1(\alpha>1)$. By Lemmas 2.1, 2.3, and 2.4, we have

$$
\begin{aligned}
\sum_{1}^{\alpha} & \equiv \sum_{n \leq x} f_{\alpha}(n) \Phi\left(\frac{x}{n}\right)=\sum_{n \leq x} \phi(n) F_{\alpha}\left(\frac{x}{n}\right) \\
& =\left(\frac{L(\alpha+1, g) x^{\alpha+1}}{\alpha+1}\right) \sum_{n \leqq x} \frac{\phi(n)}{n^{\alpha+1}}+O\left(x^{\alpha} \sum_{n \leqq x} \frac{\phi(n)}{n^{\alpha}}\right) \\
& =\left(\frac{L(\alpha+1, g) \zeta(\alpha)}{(\alpha+1) \zeta(\alpha+1)}\right) x^{\alpha+1}+O\left(x^{2}\right)+O\left(E_{\alpha}(x)\right) .
\end{aligned}
$$

Hence 


$$
\sum_{1}^{\alpha}=\left(\frac{L(\alpha+1, g) \zeta(\alpha)}{(\alpha+1) \zeta(\alpha+1)}\right) x^{\alpha+1}+O\left(E_{\alpha}(x)\right), \quad \alpha>1 .
$$

By (2.8), we also have

$$
\sum_{2}^{\alpha}=\left(\frac{L(\alpha+1, g)}{\alpha+1}\right) x^{\alpha+1}+O\left(x^{\alpha}\right),
$$

Thus (3.2) results on combining (3.5), (3.6), and (3.7). This completes the proof of Case 1.

CASE $2(\alpha=1)$. In this case, applying Lemmas 2.1 and 2.2 to $\sum_{1}^{1}$ with $z=\delta_{1}=\delta_{2}=x^{1 / 2}, g(n)=\phi(n), h(n)=f_{1}(n)$, one obtains

$$
\begin{aligned}
\sum_{1}^{1} & =\sum_{n \leq z} \phi(n) F_{1}\left(\frac{x}{n}\right)+\sum_{n \leq z} f_{1}(n) \Phi\left(\frac{x}{n}\right)-\Phi(z) F_{1}(z) \\
& \equiv \sum_{11}+\sum_{12}-\sum_{13} .
\end{aligned}
$$

On the basis of $(2.8)$ we have $(x \geqq 4)$

$$
\sum_{11}=\left(\frac{L(2, g) x^{2}}{2}\right) \sum_{n \leq z} \frac{\phi(n)}{n^{2}}+O\left(x \sum_{n \leq z} \frac{\phi(n)}{n} \log \frac{x}{n}\right) .
$$

By (2.13), or (2.10) with $f_{1}(n)=\phi(n)$, it follows that the $O$-term of $\sum_{11}$ is

$$
O\left(x \log x \sum_{n \leq x^{1 / 2}} \frac{\phi(n)}{n}\right)=O\left(x^{3 / 2} \log x\right) ;
$$

hence application of Lemma 2.4 to the first sum in $\sum_{11}$ gives

$$
\sum_{11}=\frac{L(2, g) x^{2}}{2 \zeta(2)}\left(\frac{\log x}{2}-\frac{\zeta^{\prime}(2)}{\zeta(2)}+\gamma\right)+O\left(x^{3 / 2} \log x\right) .
$$

By (2.12), one obtains

$$
\sum_{12}=\frac{x^{2}}{2 \zeta(2)} \sum_{n \leq 2} \frac{f_{1}(n)}{n^{2}}+O\left(x \sum_{n \leq 2} \frac{f_{1}(n)}{n} \log \frac{x}{n}\right) .
$$

Using (2.10), it is observed, as in the case of $\sum_{11}$, that the $O$-term of $\sum_{12}$ is $O\left(x^{3 / 2} \log x\right)$. Hence by (2.9),

(3.10) $\sum_{12}=\frac{x^{2}}{2 \zeta(2)}\left\{L(2, g)\left(\frac{\log x}{2}+\gamma\right)+L^{\prime}(2, g)\right\}+O\left(x^{3 / 2} \log x\right)$.

By (2.8) and (2.12), we have 


$$
\sum_{13}=\left(\frac{z^{2}}{2 \zeta(2)}+O(z \log z)\right)\left(\frac{L(2, g) z^{2}}{2}+O(z \log z)\right),
$$

from which it follows that

$$
\sum_{13}=\frac{L(2, g) x^{2}}{4 \zeta(2)}+O\left(x^{3 / 2} \log x\right) .
$$

Finally, by (2.8), one obtains

$$
\sum_{2}^{1}=\frac{L(2, g) x^{2}}{2}+O(x \log x) .
$$

Combining (3.5), (3.8), (3.9), (3.10), (3.11), and (3.12), the relation (3.4) results. This completes Case 2, and the theorem is proved.

REMARK. We point out the close analogy between (3.2) and the corresponding result (2.8) for functions of one variable.

The following corollary for $\sigma_{\alpha}((m, n))$ arises on placing $g(n)=1$ in the theorem.

Corollary 3.1. If $\alpha>1$, then

$$
\sum_{a, b \leq x} \sigma_{\alpha}((a, b))=\frac{x^{\alpha+1}}{\alpha+1}(2 \zeta(\alpha)-\zeta(\alpha+1))+O\left(E_{\alpha}(x)\right),
$$

where $E_{\alpha}(x)$ is defined as in (-3.3);

$$
\sum_{a, b \leq x} \sigma((a, b))=x^{2}\left(\log x+2 \gamma-\frac{1}{2}-\frac{\zeta(2)}{2}\right)+O\left(x^{3 / 2} \log x\right) .
$$

The case $g(n)=\mu(n)$ leads to the following result for $\phi_{\alpha}((m, n))$.

Corollary 3.2. If $\alpha>1$, then

$$
\sum_{a, b \leqq x} \phi_{\alpha}((a, b))
$$

$$
=\frac{x^{\alpha+1}}{(\alpha+1) \zeta^{2}(\alpha+1)}(2 \zeta(\alpha)-\zeta(\alpha+1))+O\left(E_{\alpha}(x)\right),
$$

where $E_{\alpha}(x)$ is defined by (3.3);

$$
\sum_{a, b \leq x} \phi((a, b))
$$

$$
=\frac{x^{2}}{\zeta^{2}(2)}\left(\log x+2 \gamma-\frac{1}{2}-\frac{\zeta(2)}{2}-\frac{2 \zeta^{\prime}(2)}{\zeta(2)}\right)+O\left(x^{3 / 2} \log x\right) .
$$




\title{
BIBLIOGRAPHY
}

1. Paul Bachmann, Die analytische Zahlentheorie, Leipzig, 1894.

2. Ernest Cesàro, Étude moyenne du plus grand commun diviseur de deux nombres, Ann. Mat. Pura Appl. (2) vol. 13 (1885) pp. 233-268.

3. Leonard Eugene Dickson, History of the theory of numbers, vol. II, reprinted New York, 1952.

4. J. Franel, Sur une formule utile dans la détermination de certaines valeurs asymptotiques, Math. Ann. vol. 51 (1899) pp. 369-387.

5. J. J. Sylvester, Sur le nombre de fractions ordinaires inégales qu'on peut exprimer en se servant de chiffres qui n'excèdent pas un nombre donné, C. R. Acad. Sci. Paris vol. 96 (1883) p. 409.

University OF TENNESSEE

\section{ISOTOPY IN 3-MANIFOLDS. III. CONNECTIVITY OF SPACES OF HOMEOMORPHISMS}

\author{
D. E. SANDERSON
}

1. Introduction. J. H. Roberts has reported a proof that the space $H(P)$ of homeomorphisms of the plane onto itself has exactly two components [7]. The corresponding result for three-space is proved in this paper (Roberts stated in an indirect communication to the author that he has carried out investigations along this line but does not intend to publish them). M. K. Fort proved that $H(P)$ is locally arcwise connected [4] and Fort and E. E. Floyd published a paper in which they proved that the space of homeomorphisms of the 2-sphere onto itself is uniformly locally connected [3]. Both of these results are likewise extended in this paper to the three-dimensional analogues.

2. Definitions. All of the above-mentioned results are for the compact-open topology introduced by R. H. Fox [5]. If $d(x, y)$ denotes the usual metric for Euclidean 3-space, $E^{3}$, and $C_{r}$ is the cube in $E^{3}$ with center at the origin and side $r$ (faces parallel to a fixed set of coordinate planes), then an admissible metric for the space, $H(E)$, of homeomorphisms of $E^{3}$ onto itself under the compact-open topology is $\rho(f, g)=\inf _{r>0} \max \left[1 / r, \sup _{x \in C_{r}} d(f(x), g(x))\right]$. Similarly, an admissible metric for the space, $H(S)$, of homeomorphisms of the 3sphere, $S^{3}$, onto itself is $\rho(f, g)=\sup _{x \in S^{3}} d(f(x), g(x))$. Since the treat-

Presented to the Society, May 1, 1954 under the title On isotopy of homeomorphisms $E^{8}$ and $S^{3}$; received by the editors May 15, 1959. 